\title{
EVALUTION OF GROUNDWATER QUALITY AND ITS SUITABILITY FOR DRINKING PURPOSE IN BUDHGAON, KAVALAPUR AND KARNAL VILLAGES OF SANGLI DISTRICT, MAHARASHTRA, INDIA
}

\author{
P.T.Patil ${ }^{1}$, A.S.Yadav', S. S. Jadhav ${ }^{3}$ \\ ${ }^{I}$ PVPIT, Budhgaon-416304 (M. S.), India \\ ${ }^{2}$ Dr.J.J.Magdum College of Engineering, Jaysingpur- 416101(M. S.),India \\ ${ }^{3}$ PVPIT, Budhgaon-416304(M.S.), India
}

\begin{abstract}
Groundwater is essential for any life support system. It is not only basic need for human existence but also a vital input for all development activities. The present hydro-geochemical study was confined to Budhgaon, Kavalapur and Karnal villages of Sangli district, Maharashtra (Lat.16'52'30' $N$ to $16^{0} 54^{\prime} 30^{\prime \prime} N$ and Long.74'33'45' E to 74'37'30' E). Groundwater quality and its suitability for drinking purpose were examined by physico-chemical analysis of 56 dug well water samples. These parameters were used to assess the suitability of groundwater for drinking purpose by comparing with the WHO and ISI. These analyses reveals that the groundwater is not entirely fit for drinking with respect to Hardness, EC, Ca, Cl, Bicarbonates, Sodium and Sulphate. The physico-chemical analysis of these samples reveals that $98 \%$ groundwater samples of pre-monsoon season and $70 \%$ groundwater samples of post-monsoon season represents $\mathrm{Na}+\mathrm{K}>\mathrm{Ca}+\mathrm{Mg}($ alkalies exceed alkaline earth) hydrochemicalfacies. Similarly, 100\% groundwater samples of pre-monsoon season and $84 \%$ groundwater samples of postmonsoon season reveals $\mathrm{Cl}+\mathrm{SO}_{4}>\mathrm{HCO}_{3}+\mathrm{CO}_{3}$ (strong acids exceed weak acid) hydrochemicalfacies. The 2\% and 30\% groundwater samples of pre-monsoon and post-monsoon seasons suggests $\mathrm{Ca}+\mathrm{Mg}>\mathrm{Na}+\mathrm{K}$ (alkaline earths exceed alkalies) hydrochemicalfacies respectively. The $16 \%$ groundwater samples of post-monsoon season represent $\mathrm{Cl}+\mathrm{SO}_{4}($ strong acids) hydrochemicalfacies. The chemistry of groundwater samples belongs to evaporation dominance (46\%) and rock dominance (54\%) of pre-monsoon season and evaporation dominance (64\%) and rock dominance (36\%) of post-monsoon season.
\end{abstract}

Keywords: Groundwater Quality, Sangli District, Hydro Chemical Facies.

\section{INTRODUCTION}

Water is essential to the existence of man and all leaving things. Groundwater occurs beneath the earth surface not only in single widespread aquifer but also in thousands of local aquifer systems. The quality of water is of great importance as it is commonly consumed and used by households. Rapid urbanization especially in developing countries like India has been affected the availability and quality of groundwater. The quality of groundwater is also varies with depth of water table, seasonal changes and composition of dissolved salts depending upon the sources of the salt and subsurface environment. Intensively irrigated agricultural discharges into the groundwater bring about considerable changes in the groundwater quality.

Groundwater chemistry in turn, depends on a number of factors, such as general geology, degree of chemical weathering, quality of recharge water and inputs fromsources other than water rock interaction. Such factors and their interactions result in a complex ground water quality.Panaskar et.al., 2007 [1], Yadav et.al., 2011[2], Krishna Anantha et.al., 2012 [3], Deshpande and Aher, 2012 [4], Narsimha and Anitha, 2013[5] and Pisal and Yadav,
2014 [6] have worked on the chemical aspects of groundwater from urban areas. The assessment of groundwater quality and its suitability for drinking is the objective of the present study by comparing the results against water quality standards laid down by the World Health Organization [7] and Indian Standards Institution [8].

\section{STUDY AREA}

The present study area is lies between Latitude 160 52'30" to $16054^{\prime} 30 " \mathrm{~N}$ and Longitude $74033^{\prime} 45^{\prime \prime} \mathrm{E}$ to 740 37 '30'E in Survey of India Toposheet number $47 \mathrm{~L} / 9$ is covering an area of about $62 \mathrm{~km} 2$ (Fig.1). The study area is covered by Deccan Volcanic basalt ofupper cretaceous to lower Eocene age. The average rainfall of study area is about $580 \mathrm{~mm}$. Krishna river is flowing from almost north to south in western boundary of the study area. The study area is more or less flat, having $100 \%$ irrigated land and receives the maximum water from dug wells.

\section{MATERIALS AND METHODS}

The groundwater samples were collected from 56 dug wells during pre and post-monsoon seasons of year 2013(Fig 2) 


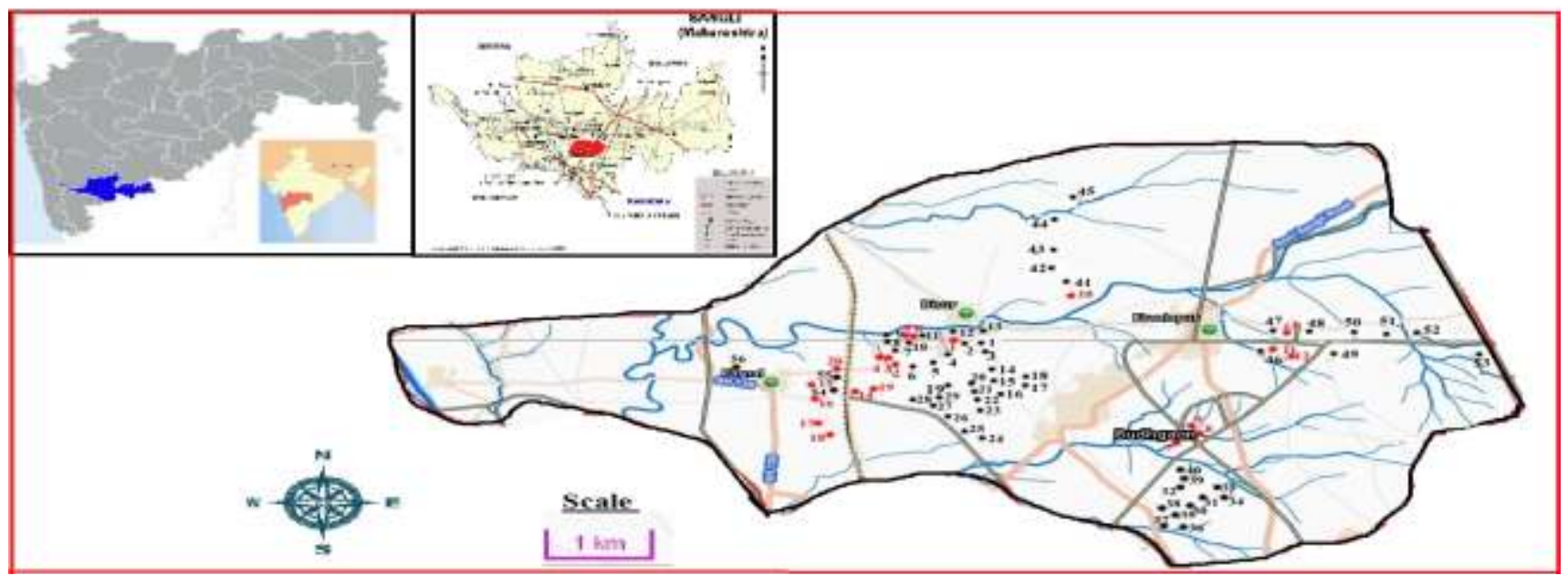

Fig. 1: Location map of study area.

The polythene bottles were used for sample collection. The different physical parameters like colour, odour, taste, foam and turbidity were measured at the time of collection of samples. The various chemical parameters were determined by using standard methods [9] (Table 1 and 2). The color, odor, taste and foam properties of dug well water samples were measured in the field. The turbidity is determined by selecting the calibration graph for the desired range and by placing the filter frame in position. Samples having the turbidity higher than $150 \mathrm{mg} / \mathrm{L}$ are tested by diluting the sample with water of very low turbidity and multiplied the result by dilution factor. The $\mathrm{P}^{\mathrm{H}}$ and Electrical Conductivity (EC) were measured with the help of $\mathrm{P}^{\mathrm{H}}$ meter and Conductivity meter respectively. The chemical parameters viz; Total Hardness( $\mathrm{TH})$, Calcium $(\mathrm{Ca})$, Magnesium( $\mathrm{Mg})$, Bicarbonates $\left(\mathrm{HCO}_{3}\right)$, Carbonates $\left(\mathrm{CO}_{3}\right)$ and Chlorides $(\mathrm{Cl})$ were determined by titration. The Sodium (Na) and Potassium (K) were found out by using Flame photometer. Sulphate $\left(\mathrm{SO}_{4}\right)$ was determined by using UV-Visible Spectrometer. The Total Dissolved Solids (TDS) were calculated by multiplying EC values with 0.64 [10].

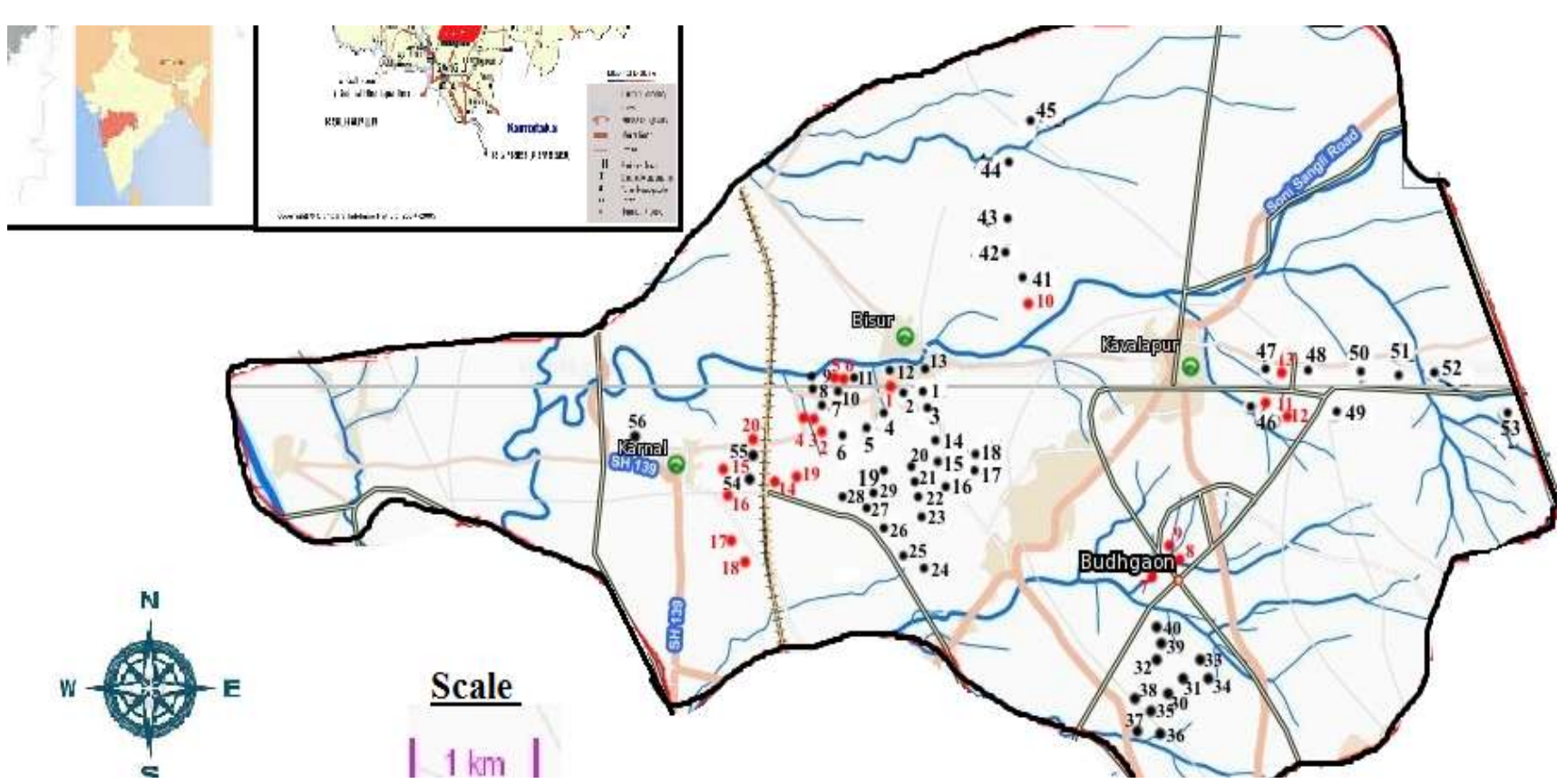

Fig. 2: Sample Location map of study area.

\subsection{Groundwater Properties}

\subsection{1. $P^{H}$}

The relative concentration of hydrogen ions in water indicates whether the water is acidic or alkaline in nature. $\mathrm{P}^{\mathrm{H}}$ of dug well water samples varies from 6.11 to 8.00 for pre- monsoon season and in the post-monsoon season it varies from 6.78 to 8.25 . The $\mathrm{P}^{\mathrm{H}}$ of $80 \%$ water samples in premonsoon season and $91 \%$ in post-monsoon season ranges from 6.5 to 7.5. This shows that there is very little seasonal fluctuation of $\mathrm{P}^{\mathrm{H}}$ values in the region. 
Table 1: Concentration of different chemical parameters of bore well water of the study area (pre-monsoon season).

\begin{tabular}{|c|c|c|c|c|c|c|c|c|c|c|c|c|}
\hline \multirow{2}{*}{$\begin{array}{l}\text { Parameters/ } \\
\text { Sample } \\
\text { nos. }\end{array}$} & \multirow[b]{2}{*}{$\mathrm{P}^{\mathrm{H}}$} & \multirow{2}{*}{$\begin{array}{l}\mathrm{EC} \\
(\mu / \mathrm{cm})\end{array}$} & $\mathrm{TH}$ & TDS & $\mathrm{Ca}$ & $\mathrm{Mg}$ & $\mathrm{Na}$ & $\mathrm{K}$ & $\mathrm{HCO}_{3}$ & $\mathrm{CO}_{3}$ & $\mathrm{SO}_{4}$ & $\mathrm{Cl}$ \\
\hline & & & \multicolumn{10}{|c|}{$\mathrm{mg} / \mathrm{L}$} \\
\hline DW 1 & 7.11 & 1580 & 390 & 990 & 205.0 & 17.25 & 365.01 & 14.82 & 280.60 & 0 & 672.96 & 330.15 \\
\hline$" 2$ & 6.81 & 2930 & 450 & 1760 & 245.0 & 18.10 & 470.12 & 9.75 & 427.00 & 0 & 786.24 & 401.15 \\
\hline$" 3$ & 7.28 & 1440 & 410 & 1260 & 135.0 & 20.05 & 255.07 & 4.68 & 280.60 & 0 & 355.68 & 269.80 \\
\hline$" 4$ & 7.35 & 850 & 360 & 2390 & 105.0 & 11.30 & 195.04 & 4.68 & 231.80 & 0 & 215.04 & 230.75 \\
\hline$" 5$ & 7.62 & 320 & 380 & 1820 & 60.0 & 13.24 & 155.02 & 4.68 & 134.20 & 0 & 204.00 & 159.75 \\
\hline$" 6$ & 7.19 & 3390 & 610 & 2760 & 155.0 & 24.18 & 310.04 & 9.75 & 475.80 & 0 & 132.96 & 457.95 \\
\hline$" 7$ & 7.11 & 3250 & 630 & 2580 & 165.0 & 24.30 & 320.16 & 9.75 & 427.00 & 0 & 255.36 & 429.55 \\
\hline$" 8$ & 7.12 & 1850 & 410 & 1960 & 105.0 & 15.19 & 205.16 & 4.68 & 256.20 & 0 & 150.72 & 273.35 \\
\hline$" 9$ & 7.92 & 3560 & 640 & 2260 & 155.0 & 22.48 & 357.42 & 14.82 & 378.20 & 0 & 322.56 & 443.75 \\
\hline$" 10$ & 6.9 & 3580 & 610 & 1890 & 195.0 & 26.37 & 365.01 & 4.68 & 463.60 & 0 & 298.08 & 500.55 \\
\hline$" 11$ & 6.65 & 6360 & 910 & 2990 & 295.0 & 30.38 & 755.32 & 24.96 & 866.20 & 0 & 443.04 & 969.15 \\
\hline$" 12$ & 7.17 & 4700 & 810 & 2860 & 245.0 & 28.43 & 405.03 & 19.89 & 500.20 & 0 & 465.60 & 607.05 \\
\hline$" 13$ & 6.65 & 6380 & 920 & 2960 & 305.0 & 32.20 & 905.28 & 102.96 & 841.80 & 0 & 902.40 & 898.15 \\
\hline$" 14$ & 7.76 & 1790 & 390 & 1220 & 155.0 & 13.24 & 160.08 & 4.68 & 256.20 & 0 & 212.16 & 259.15 \\
\hline$" 15$ & 7.37 & 1860 & 495 & 1860 & 169.0 & 16.52 & 444.36 & 16.38 & 259.86 & 0 & 907.20 & 277.96 \\
\hline$" 16$ & 7.2 & 1900 & 470 & 890 & 62.4 & 15.31 & 433.78 & 43.68 & 263.52 & 0 & 716.16 & 269.44 \\
\hline$" 17$ & 7.11 & 1820 & 420 & 1260 & 135.0 & 15.55 & 244.95 & 9.75 & 256.20 & 0 & 356.64 & 259.15 \\
\hline$" 18$ & 7.36 & 760 & 360 & 1380 & 105.0 & 12.88 & 155.02 & 4.68 & 146.40 & 0 & 263.52 & 188.15 \\
\hline$" 19$ & 7.7 & 1400 & 500 & 1930 & 272.0 & 20.29 & 705.18 & 19.89 & 494.10 & 0 & 1146.24 & 571.55 \\
\hline$" 20$ & 7 & 1400 & 292 & 1860 & 164.0 & 19.32 & 760.38 & 19.89 & 634.40 & 0 & 1075.68 & 749.05 \\
\hline$" 21$ & 7.6 & 1400 & 295 & 1950 & 328.0 & 24.18 & 1161.50 & 29.64 & 1122.40 & 0 & 1515.84 & 781.00 \\
\hline$" 22$ & 7.4 & 1400 & 410 & 2680 & 270.0 & 24.30 & 1270.52 & 24.96 & 1085.80 & 0 & 1513.44 & 1111.15 \\
\hline$" 23$ & 7.4 & 1400 & 380 & 1760 & 322.0 & 19.32 & 755.32 & 34.71 & 628.30 & 0 & 1184.16 & 685.15 \\
\hline$" 24$ & 7.3 & 1410 & 474 & 1960 & 167.2 & 20.90 & 753.25 & 29.25 & 713.70 & 0 & 1179.36 & 784.55 \\
\hline$" 25$ & 7.8 & 1408 & 386 & 1820 & 118.0 & 19.93 & 683.56 & 25.35 & 817.40 & 0 & 1128.00 & 766.80 \\
\hline$" 26$ & 8 & 1396 & 520 & 980 & 204.0 & 18.59 & 725.42 & 23.01 & 927.20 & 0 & 1204.80 & 759.70 \\
\hline "27 & 8 & 1408 & 450 & 2640 & 298.0 & 20.78 & 706.10 & 24.96 & 878.40 & 0 & 1199.04 & 635.45 \\
\hline$" 28$ & 7.6 & 1415 & 410 & 3600 & 323.6 & 19.68 & 673.90 & 17.55 & 707.60 & 0 & 1075.20 & 702.90 \\
\hline$" 29$ & 7.2 & 1408 & 360 & 1960 & 382.4 & 19.20 & 747.04 & 24.18 & 756.40 & 0 & 1027.20 & 678.05 \\
\hline$" 30$ & 7.16 & 4680 & 750 & 1860 & 305.0 & 20.29 & 705.18 & 19.89 & 494.10 & 0 & 1146.24 & 571.55 \\
\hline , 31 & 6.98 & 5310 & 820 & 2740 & 385.0 & 21.63 & 770.04 & 25.74 & 744.81 & 0 & 1259.04 & 1535.02 \\
\hline$" 32$ & 6.75 & 8350 & 810 & 2160 & 455.0 & 24.30 & 1270.52 & 24.96 & 1085.80 & 0 & 1513.44 & 1111.15 \\
\hline$" 33$ & 6.51 & 5180 & 790 & 3820 & 355.0 & 19.32 & 760.38 & 19.89 & 634.40 & 0 & 1075.68 & 749.05 \\
\hline$" \quad 34$ & 6.62 & 6400 & 805 & 1960 & 392.0 & 24.06 & 1020.74 & 23.01 & 732.00 & 0 & 1210.56 & 1019.91 \\
\hline$" 35$ & 6.58 & 6200 & 900 & 2860 & 384.2 & 20.29 & 719.21 & 25.74 & 868.64 & 0 & 1647.36 & 860.87 \\
\hline$" 36$ & 5.49 & 4860 & 780 & 3140 & 385.0 & 17.98 & 686.32 & 33.15 & 625.25 & 0 & 1088.64 & 648.23 \\
\hline$" 37$ & 6.65 & 7860 & 890 & 1860 & 453.8 & 22.96 & 1362.52 & 33.93 & 1171.81 & 0 & 1569.12 & 1180.02 \\
\hline$" 38$ & 6.51 & 5580 & 815 & 2760 & 424.4 & 21.63 & 695.29 & 30.42 & 746.64 & 0 & 1375.68 & 767.86 \\
\hline$" 39$ & 6.65 & 4850 & 820 & 2930 & 375.0 & 19.32 & 755.32 & 34.71 & 622.20 & 0 & 1184.16 & 685.15 \\
\hline$" 40$ & 6.65 & 4850 & 820 & 2960 & 375.0 & 19.32 & 755.32 & 34.71 & 622.20 & 0 & 1184.16 & 685.15 \\
\hline$" 41$ & 7.14 & 6040 & 910 & 3100 & 305.0 & 30.38 & 787.29 & 19.89 & 719.80 & 0 & 947.04 & 770.35 \\
\hline$" 42$ & 6.87 & 7480 & 980 & 3120 & 345.0 & 32.20 & 885.27 & 29.64 & 841.80 & 0 & 1000.80 & 855.55 \\
\hline$" 43$ & 7.29 & 7350 & 960 & 4920 & 335.0 & 31.23 & 945.30 & 34.71 & 866.20 & 0 & 950.88 & 969.15 \\
\hline$" 44$ & 7.14 & 6430 & 810 & 4890 & 295.0 & 29.40 & 655.27 & 24.96 & 707.60 & 0 & 643.20 & 756.15 \\
\hline$" 45$ & 7.13 & 6100 & 830 & 4020 & 265.0 & 29.16 & 695.42 & 29.64 & 719.80 & 0 & 630.72 & 770.35 \\
\hline$" 46$ & 6.92 & 5160 & 710 & 4120 & 295.0 & 22.11 & 695.29 & 24.96 & 866.20 & 0 & 649.92 & 969.15 \\
\hline$" 47$ & 7.26 & 1340 & 520 & 3160 & 105.0 & 13.24 & 204.93 & 4.68 & 256.20 & 0 & 185.76 & 259.15 \\
\hline$" 48$ & 7.35 & 1960 & 490 & 1980 & 110.0 & 14.22 & 220.11 & 9.75 & 268.40 & 0 & 191.52 & 287.55 \\
\hline$" 49$ & 7.2 & 3850 & 620 & 2760 & 245.0 & 19.08 & 455.17 & 22.62 & 305.00 & 0 & 945.12 & 330.15 \\
\hline$" 50$ & 7.11 & 10040 & 990 & 3910 & 560.0 & 32.20 & 1555.72 & 34.71 & 1256.60 & 0 & 1703.04 & 1423.55 \\
\hline$" 51$ & 6.94 & 7890 & 910 & 6920 & 295.0 & 21.26 & 855.37 & 24.96 & 768.60 & 0 & 356.64 & 855.55 \\
\hline$" 52$ & 6.11 & 7470 & 890 & 6120 & 235.0 & 22.48 & 895.39 & 24.96 & 817.40 & 0 & 732.96 & 869.75 \\
\hline Parameters/ & $\mathrm{P}^{\mathrm{H}}$ & $\mathrm{EC}$ & $\mathrm{TH}$ & TDS & $\mathrm{Ca}$ & $\mathrm{Mg}$ & $\mathrm{Na}$ & $\mathrm{K}$ & $\mathrm{HCO}_{3}$ & $\mathrm{CO}_{3}$ & $\mathrm{SO}_{4}$ & $\mathrm{Cl}$ \\
\hline
\end{tabular}




\begin{tabular}{|c|c|c|c|c|c|c|c|c|c|c|c|c|}
\hline $\begin{array}{l}\text { Sample } \\
\text { nos. }\end{array}$ & & $(\mu / \mathrm{cm})$ & \multicolumn{10}{|c|}{$\mathrm{mg} / \mathrm{L} \longleftarrow$} \\
\hline$" 53$ & 6.99 & 7650 & 920 & 3960 & 240.0 & 23.57 & 905.28 & 19.89 & 829.60 & 0 & 2462.40 & 883.95 \\
\hline$" 54$ & 7.12 & 1500 & 390 & 3720 & 115.0 & 13.00 & 255.07 & 9.75 & 353.80 & 0 & 127.68 & 344.35 \\
\hline$" 55$ & 8.81 & 2450 & 420 & 1620 & 195.0 & 15.07 & 295.09 & 9.75 & 414.80 & 0 & 107.52 & 528.95 \\
\hline$" \quad 56$ & 7.24 & 2580 & 440 & 1980 & 155.0 & 19.32 & 620.08 & 9.75 & 549.00 & 0 & 511.68 & 599.95 \\
\hline
\end{tabular}

Table 2: Concentration of different chemical parameters of bore well water of the study area (post-monsoon season).

\begin{tabular}{|c|c|c|c|c|c|c|c|c|c|c|c|c|}
\hline \multirow{2}{*}{$\begin{array}{l}\text { Parameters/ } \\
\text { Sample } \\
\text { nos. }\end{array}$} & \multirow{2}{*}{$\mathrm{P}^{\mathrm{H}}$} & \multirow{2}{*}{$\begin{array}{l}\text { EC } \\
(\mu / \mathrm{cm})\end{array}$} & $\mathrm{TH}$ & TDS & $\mathrm{Ca}$ & $\mathrm{Mg}$ & $\mathrm{Na}$ & $\mathrm{K}$ & $\mathrm{HCO}_{3}$ & $\mathrm{CO}_{3}$ & $\mathrm{SO}_{4}$ & $\mathrm{Cl}$ \\
\hline & & & \multicolumn{10}{|c|}{$\mathrm{mg} / \mathrm{L} \longleftarrow$} \\
\hline DW 1 & 7.14 & 1620 & 410 & 980 & 75 & 21.38 & 810.52 & 5.07 & 353.8 & 0 & 1308.96 & 259.15 \\
\hline$" 2$ & 6.87 & 3460 & 650 & 2190 & 65 & 32.32 & 815.35 & 30.03 & 378.2 & 0 & 1201.44 & 113.25 \\
\hline$" 3$ & 7.21 & 3020 & 620 & $11 ` 90$ & 70 & 30.5 & 705.41 & 14.82 & 353.8 & 0 & 1111.68 & 287.55 \\
\hline$" 4$ & 6.95 & 3690 & 790 & 3340 & 120 & 27.46 & 555.22 & 24.96 & 436.76 & 0 & 525.12 & 502.33 \\
\hline$" 5$ & 7.27 & 4370 & 710 & 2750 & 215 & 18.35 & 775.33 & 24.96 & 475.8 & 0 & 1262.88 & 431.33 \\
\hline$" 6$ & 7.16 & 4520 & 810 & 2860 & 245 & 25.39 & 825.47 & 19.89 & 488 & 0 & 1509.12 & 401.15 \\
\hline " 7 & 7.2 & 4160 & 820 & 2630 & 200 & 36.57 & 655.27 & 14.82 & 500.2 & 0 & 1055.04 & 396.54 \\
\hline$" 8$ & 7.12 & 2760 & 420 & 1750 & 180 & 32.56 & 475.18 & 35.1 & 256.2 & 0 & 1043.04 & 259.15 \\
\hline$" 9$ & 7.38 & 4480 & 530 & 2780 & 180 & 32.32 & 785.45 & 30.03 & 500.2 & 0 & 1260.96 & 429.55 \\
\hline$" 10$ & 7.26 & 4210 & 626 & 2160 & 196 & 28.67 & 686.78 & 30.42 & 524.6 & 0 & 1349.76 & 421.03 \\
\hline$" 11$ & 7.18 & 5180 & 990 & 3250 & 240 & 35.96 & 756.7 & 30.03 & 512.4 & 0 & 1335.36 & 396.54 \\
\hline$" 12$ & 6.95 & 6940 & 990 & 3250 & 250 & 37.06 & 1840.92 & 35.1 & 500.2 & 0 & 3691.2 & 429.55 \\
\hline$" 13$ & 6.9 & 5920 & 995 & 3260 & 298 & 30.62 & 234.6 & 33.54 & 394.06 & 0 & 946.56 & 422.45 \\
\hline$" 14$ & 7.56 & 2710 & 590 & 2000 & 170 & 26 & 190.67 & 28.08 & 361.12 & 0 & 488.64 & 344.35 \\
\hline$" 15$ & 7.13 & 2610 & 490 & 1670 & 160 & 22.36 & 420.21 & 19.89 & 256.2 & 0 & 821.76 & 259.15 \\
\hline$" 16$ & 6.89 & 1360 & 480 & 890 & 50 & 21.38 & 395.14 & 54.99 & 256.2 & 0 & 544.8 & 259.15 \\
\hline$" 17$ & 7.18 & 3080 & 490 & 1500 & 205 & 26.24 & 265.19 & 40.95 & 378.2 & 0 & 42.24 & 361.75 \\
\hline$" 18$ & 7.24 & 2150 & 410 & 1420 & 175 & 25.39 & 275.08 & 10.14 & 374.54 & 0 & 295.68 & 111.83 \\
\hline$" 19$ & 7.23 & 3750 & 510 & 2430 & 290 & 28.31 & 590.41 & 35.1 & 402.6 & 0 & 1223.52 & 401.15 \\
\hline$" 20$ & 7.22 & 3330 & 530 & 2190 & 160 & 27.22 & 630.43 & 44.85 & 378.2 & 0 & 1159.2 & 287.55 \\
\hline$" 21$ & 7.17 & 3550 & 590 & 2320 & 310 & 30.25 & 410.32 & 30.03 & 427 & 0 & 921.12 & 325.54 \\
\hline$" 22$ & 7.15 & 5200 & 990 & 3380 & 280 & 32.32 & 885.5 & 19.89 & 475.8 & 0 & 1745.76 & 401.15 \\
\hline$" 23$ & 6.92 & 5080 & 990 & 2320 & 300 & 31.35 & 710.47 & 5.07 & 500.2 & 0 & 1357.92 & 145.55 \\
\hline$" 24$ & 7.22 & 4980 & 788 & 3260 & 190 & 28.43 & 525.32 & 5.07 & 475.8 & 0 & 907.68 & 287.55 \\
\hline$" 25$ & 7.46 & 3680 & 610 & 2420 & 130 & 27.22 & 730.48 & 10.14 & 390.4 & 0 & 1144.8 & 360.33 \\
\hline$" 26$ & 8.25 & 1460 & 390 & 980 & 185 & 21.38 & 535.21 & 5.07 & 256.2 & 0 & 1118.88 & 219.75 \\
\hline$" 27$ & 7.14 & 4680 & 890 & 3090 & 310 & 29.52 & 985.55 & 10.14 & 436.76 & 0 & 2137.44 & 315.95 \\
\hline$" 28$ & 7.14 & 4380 & 910 & 2190 & 355 & 39.61 & 570.4 & 39.78 & 475.8 & 0 & 2291.04 & 401.15 \\
\hline$" 29$ & 7.22 & 3250 & 710 & 2150 & 380 & 28.43 & 255.07 & 14.82 & 378.2 & 0 & 871.68 & 287.55 \\
\hline$" 30$ & 7.1 & 4290 & 820 & 2830 & 315 & 33.78 & 302.91 & 30.03 & 475.8 & 0 & 776.16 & 301.75 \\
\hline, 31 & 7.16 & 5260 & 990 & 3460 & 500 & 29.65 & 422.51 & 35.1 & 488 & 0 & 1255.2 & 429.55 \\
\hline$" \quad 32$ & 6.92 & 7550 & 990 & 5030 & 450 & 36.33 & 795.34 & 44.85 & 597.8 & 0 & 1887.84 & 429.55 \\
\hline$" 33$ & 7.19 & 4520 & 890 & 2980 & 495 & 37.42 & 560.28 & 30.03 & 475.8 & 0 & 1778.4 & 287.55 \\
\hline$" 34$ & 7.71 & 4280 & 850 & 2800 & 365 & 36.57 & 610.42 & 39.78 & 436.76 & 0 & 1571.04 & 301.75 \\
\hline$" 35$ & 7.16 & 6840 & 990 & 4500 & 500 & 38.39 & 500.25 & 10.14 & 378.2 & 0 & 1567.68 & 395.12 \\
\hline$" 36$ & 7.17 & 4020 & 830 & 2640 & 505 & 38.64 & 435.16 & 24.96 & 390.4 & 0 & 1607.52 & 394.05 \\
\hline$" 37$ & 6.99 & 6030 & 990 & 3940 & 510 & 39.37 & 595.24 & 10.14 & 500.2 & 0 & 1698.24 & 401.15 \\
\hline$" 38$ & 7.10 & 6130 & 990 & 4000 & 515 & 34.51 & 640.32 & 30.03 & 512.4 & 0 & 1780.8 & 396.54 \\
\hline$" 39$ & 7.15 & 6300 & 990 & 4280 & 595 & 30.38 & 670.45 & 24.96 & 500.2 & 0 & 2021.28 & 401.15 \\
\hline$" 40$ & 7.11 & 6750 & 990 & 4520 & 410 & 31.47 & 1003.49 & 24.96 & 475.8 & 0 & 2422.08 & 287.55 \\
\hline$" 41$ & 6.82 & 7100 & 990 & 4800 & 480 & 39.61 & 570.4 & 24.96 & 475.8 & 0 & 1765.92 & 287.55 \\
\hline$" 42$ & 6.69 & 8140 & 990 & 5520 & 1700 & 33.29 & 3026.57 & 24.96 & 500.2 & 0 & 9430.56 & 539.6 \\
\hline$" 43$ & 6.9 & 8030 & 990 & 5360 & 1780 & 38.64 & 3001.5 & 24.96 & 524.6 & 0 & 9513.6 & 571.55 \\
\hline$" 44$ & 6.95 & 7580 & 990 & 5050 & 5 & 31.35 & 1210.72 & 44.85 & 549 & 0 & 4193.28 & 573.33 \\
\hline \multirow{2}{*}{$\begin{array}{l}\text { Parameters/ } \\
\text { Sample } \\
\text { nos. }\end{array}$} & \multirow[t]{2}{*}{$\mathrm{P}^{\mathrm{H}}$} & $\begin{array}{l}\text { EC } \\
(\mu / \mathrm{cm})\end{array}$ & $\mathrm{TH}$ & TDS & $\mathrm{Ca}$ & $\mathrm{Mg}$ & $\mathrm{Na}$ & $\mathrm{K}$ & $\mathrm{HCO}_{3}$ & $\mathrm{CO}_{3}$ & $\mathrm{SO}_{4}$ & $\mathrm{Cl}$ \\
\hline & & & \multicolumn{10}{|c|}{ meft } \\
\hline
\end{tabular}




\begin{tabular}{|c|c|c|c|c|c|c|c|c|c|c|c|c|c|}
\hline$"$ & 45 & 6.78 & 6850 & 990 & 4580 & 1130 & 31.47 & 1255.57 & 30.03 & 378.2 & 0 & 4184.16 & 401.15 \\
\hline$"$ & 46 & 6.99 & 6560 & 990 & 4350 & 860 & 29.52 & 1000.5 & 30.03 & 475.8 & 0 & 3205.92 & 429.55 \\
\hline 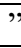 & 47 & 7.30 & 1520 & 510 & 790 & 800 & 30.25 & 546.25 & 39.78 & 256.2 & 0 & 1573.92 & 259.15 \\
\hline$"$ & 48 & 6.88 & 2920 & 590 & 1950 & 350 & 24.42 & 515.2 & 39.78 & 268.4 & 0 & 1100.64 & 287.55 \\
\hline$"$ & 49 & 6.88 & 4340 & 810 & 2870 & 300 & 30.25 & 620.31 & 10.14 & 390.4 & 0 & 2196.96 & 330.15 \\
\hline$"$ & 50 & 7.69 & 6080 & 990 & 3930 & 635 & 39.61 & 1050.64 & 44.85 & 622.2 & 0 & 2905.92 & 578.65 \\
\hline$"$ & 51 & 7.11 & 11390 & 990 & 7580 & 735 & 39.85 & 1600.8 & 49.92 & 646.6 & 0 & 4128.48 & 685.15 \\
\hline$"$ & 52 & 7.14 & 9080 & 990 & 6040 & 835 & 41.43 & 865.49 & 14.82 & 719.8 & 0 & 2362.56 & 572.26 \\
\hline , & 53 & 7.31 & 6260 & 990 & 4060 & 715 & 36.57 & 678.96 & 24.96 & 475.8 & 0 & 2792.64 & 301.75 \\
\hline$"$ & 54 & 7.46 & 2050 & 610 & 1360 & 825 & 32.56 & 615.25 & 30.03 & 256.2 & 0 & 1497.6 & 259.15 \\
\hline 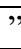 & 55 & 7.77 & 2660 & 620 & 1370 & 250 & 32.56 & 635.26 & 36.66 & 305 & 0 & 1492.8 & 287.55 \\
\hline$"$ & 56 & 7.27 & 3150 & 710 & 2050 & 260 & 33.53 & 670.45 & 36.66 & 378.2 & 0 & 2162.88 & 330.15 \\
\hline
\end{tabular}

\subsubsection{Electric Conductivity (EC)}

Electric conductivityis the measure of water capacity to convey electric current. EC of dug well samples varies from $320 \mu \mathrm{s} / \mathrm{cm}$ to $10040 \mu \mathrm{s} / \mathrm{cm}$ in pre-monsoon season and
$1360 \mu \mathrm{s} / \mathrm{cm}$ to $11390 \mu \mathrm{s} / \mathrm{cm}$ in post-monsoon season, which is very high. The EC value of $100 \%$ samples in pre and postmonsoon seasons are higher than permissible limit (Table $3)$.

Table 3: Classification of groundwater on the basis of Electric conductivity values

\begin{tabular}{|l|l|l|l|l|l|}
\hline \multirow{2}{*}{$\begin{array}{l}\text { Conductivity range } \\
(\mu \mathrm{s} / \mathrm{cm})\end{array}$} & \multirow{2}{*}{ Classification } & \multicolumn{2}{l|}{ Pre-monsoon Season } & \multicolumn{2}{l|}{ Post-monsoon Season } \\
\cline { 3 - 6 } & & No. of samples & $\%$ & No. of samples & $\%$ \\
\hline$<1500$ & Permissible & 16 & 28 & 03 & 5 \\
\hline $1500-3000$ & Not permissible & 11 & 20 & 08 & 14 \\
\hline$>3000$ & Hazardous & 29 & 52 & 45 & 81 \\
\hline
\end{tabular}

\subsubsection{Total Dissolved Solids (TDS)}

Total dissolved solids are the total amount of mobile charged ions, including minerals, salts or metals dissolved in given volume of water, expressed in units of $\mathrm{mg} / \mathrm{lit}$. The TDS of dug well samples in study area varies from 890 $\mathrm{mg} / \mathrm{lit}$ to $6920 \mathrm{mg} / \mathrm{lit}$ in pre-monsoon season and $790 \mathrm{mg} / \mathrm{lit}$ to $7580 \mathrm{mg} / \mathrm{lit}$ in post-monsoon season. The higher value of TDS is attributed to application of agricultural fertilizer contributing the higher concentration of ions in to the groundwater (Table 4). The 95\% samples are exceeding maximum permissible limit.

Table 4: Classification of groundwater on the basis TDS values

\begin{tabular}{|l|l|l|l|l|l|}
\hline \multirow{2}{*}{ TDS } & \multirow{2}{*}{ Classification } & Pre-monsoon Season & \multicolumn{2}{l|}{ Post-monsoon Season } \\
\cline { 3 - 6 } & & No. of samples & $\%$ & No. of samples & $\%$ \\
\hline $150-300$ & Excellent & 0 & 0 & 0 & 0 \\
\hline $300-600$ & Good & 0 & 0 & 0 & 0 \\
\hline $600-900$ & Fair & 1 & 2 & 0 & 0 \\
\hline $900-1200$ & Poor & 2 & 3 & 3 & 5 \\
\hline Above 1200 & unacceptable & 53 & 95 & 53 & 95 \\
\hline
\end{tabular}

\subsubsection{Total Hardness (TH)}

The total hardness of dug well water samples in premonsoon season varies from $292 \mathrm{mg} / \mathrm{lit}$. to $990 \mathrm{mg} / \mathrm{lit}$. and $390 \mathrm{mg} / \mathrm{lit}$ to $990 \mathrm{mg} / \mathrm{lit}$. in post-monsoon season. It is seen that the total hardness of dug well water samples from post- monsoon season is higher compared to post-monsoon season. The $96 \%$ and $100 \%$ dug well water samples in premonsoon and post-monsoon seasons respectively are classified as very hard water (Table 5).

Table 5: Classification of groundwater on the basis Total Hardness values

\begin{tabular}{|l|l|l|l|l|l|}
\hline Total Hardness as & Water Class & Pre-monsoon Season & \multicolumn{2}{l|}{ Post-monsoon Season } \\
\cline { 3 - 6 } $\mathrm{CaCO}_{3}(\mathrm{mg} / \mathrm{lit})$ & & No. of samples & $\%$ & No. of samples & $\%$ \\
\hline$<75$ & Soft & - & - & - & - \\
\hline $75-150$ & Moderately Hard & - & - & - & - \\
\hline $150-300$ & Hard & 02 & $4 \%$ & - & - \\
\hline$>300$ & Very hard & 54 & $96 \%$ & 56 & $100 \%$ \\
\hline
\end{tabular}




\subsubsection{Calcium(Ca)}

Calcium is naturally present in water. Calcium is determinant of water hardness, because it can be found in water as $\mathrm{Ca}$ ions. Calcium content in dug well water samples varies from $60 \mathrm{mg} / \mathrm{lit}$ to $560 \mathrm{mg} / \mathrm{lit}$ in pre-monsoon season and $50 \mathrm{mg} /$ lit to $1780 \mathrm{mg} / \mathrm{lit}$ in post-monsoon season (Table
6).The $63 \%$ of samples from pre-monsoon season and $72 \%$ of samples from post-monsoon season exceed the maximum permissible limit. Calcium content in groundwater increases in post-monsoon season.

Table 6: Classification of groundwater on the basis Calcium values

\begin{tabular}{|l|l|l|l|l|}
\hline \multirow{2}{*}{$\mathrm{Ca}$} & Pre-monsoon Season & \multicolumn{2}{l|}{ Post-monsoon Season } \\
\cline { 2 - 5 } & No. of samples & $\%$ & No. of samples & $\%$ \\
\hline$<75$ & 2 & 3 & 4 & 7 \\
\hline $75-200$ & 19 & 34 & 12 & 21 \\
\hline$>200$ & 35 & 63 & 40 & 72 \\
\hline
\end{tabular}

\subsubsection{Magnesium (Mg)}

A large number of minerals contain magnesium. Magnesium is washed from rocks and subsequently ends up in water. Magnesium has many different purposes and consequently may end up in water in many different ways. It adds up in the environment by use of fertilizers for agricultural practice and from cattle feed. The value of magnesium from dug well water samples ranges from $11.3 \mathrm{mg} / \mathrm{lit}$. to $32.2 \mathrm{mg} / \mathrm{lit}$. in pre-monsoon season and $18.35 \mathrm{mg} / \mathrm{lit}$. to $41.43 \mathrm{mg} / \mathrm{lit}$. in post-monsoon season. The $7 \%$ groundwater samples in premonsoon season and $64 \%$ in post-monsoon season exceeds the highest desirable limit. The $30 \%$ of magnesium in postmonsoon season increases.

\subsubsection{Chloride (Cl)}

Chloride originates from sodium chloride which gets dissolved in water from rock and soil. The concentration of chloride in groundwater will increase if it is mixed with sewage or sea water. The chloride content in the study area varies from $159.40 \mathrm{mg} / \mathrm{lit}$. to $1428.54 \mathrm{mg} / \mathrm{lit}$. in premonsoon season and $113.26 \mathrm{mg} / \mathrm{lit}$. to $685.15 \mathrm{mg} / \mathrm{lit}$. in post monsoon season.

\subsubsection{Alkalinity (Carbonates and Bicarbonate)}

Alkalinity is the measure of the capacity of the water to neutralize a strong acid. The alkalinity in the water is generally imparted by the salts of carbonates, silicates etc. together with hydroxyl ions in free states. The bicarbonate alkalinity of dug well water samples varies from 146.4 $\mathrm{mg} / \mathrm{lit}$. to $1256.4 \mathrm{mg} / \mathrm{lit}$. in pre-monsoon season and 256.2 $\mathrm{mg} / \mathrm{lit}$. to $729.8 \mathrm{mg} / \mathrm{lit}$. in post-monsoon season.

\subsubsection{Sodium(Na)}

Sodium concentration in groundwater samples of study area is high as compare to $\mathrm{Ca}$ and $\mathrm{Mg}$. The sodium content in dug well water samples varies from $195.04 \mathrm{mg} / \mathrm{lit}$. to $1555.70 \mathrm{mg} / \mathrm{lit}$. in pre-monsoon season and $190.67 \mathrm{mg} / \mathrm{lit}$. to $3026.57 \mathrm{mg} / \mathrm{lit}$. in post-monsoon season.

\subsection{Classification of Groundwater based on Piper}

\section{Trilinear Diagram}

In order to understand the variation in hydro-chemical facies with space and time, the data has been plotted on the Piper Trilineardiagram [11].

It is seen from the Fig. 3.a that out of 56 dug well water samples of pre-monsoon season, 55 dug well water samples (98\%) represent $\mathrm{Na}+\mathrm{K}>\mathrm{Ca}+\mathrm{Mg}$ (alkalies exceed alkaline earth) and 1 dug well water sample(2\%) belong to $\mathrm{Ca}+\mathrm{Mg}>\mathrm{Na}+\mathrm{K}$ (alkaline earths exceed alkalies) hydrochemicalfacies. Similarly, 56 dug well water samples $(100 \%)$ suggests $\mathrm{Cl}+\mathrm{SO}_{4}>\mathrm{HCO}_{3}+\mathrm{CO}_{3}$ (strong acids exceed weak acids) hydrochemicalfacies.

From the Fig. 3.b, it is seen that out of 56 dug well water samples of post-monsoon season, 39 dug well water samples $(70 \%)$ represents $\mathrm{Na}+\mathrm{K}>\mathrm{Ca}+\mathrm{Mg}$ (alkalies exceed alkaline earth) and $17 \mathrm{dug}$ well water samples (30\%) suggests $\mathrm{Ca}+\mathrm{Mg}>\mathrm{Na}+\mathrm{K}$ (alkaline earths exceed alkalies) hydrochemicalfacies. Similarly, 47 dug well water samples $(84 \%)$ suggests $\mathrm{Cl}+\mathrm{SO}_{4}>\mathrm{HCO}_{3}+\mathrm{CO}_{3}$ (strong acids exceed weak acids) and 9 dug well water samples (16\%) represent $\mathrm{Cl}+\mathrm{SO}_{4}$ (strong acids) hydrochemicalfacies. 


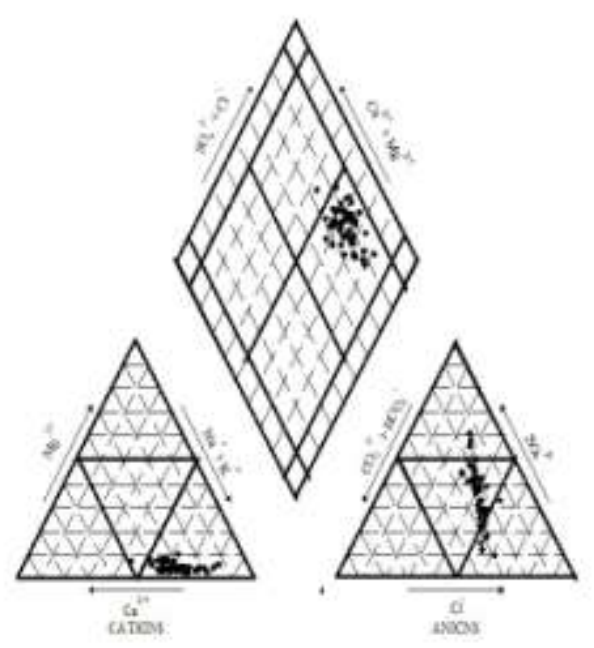

(a. Pre-monsoon season)

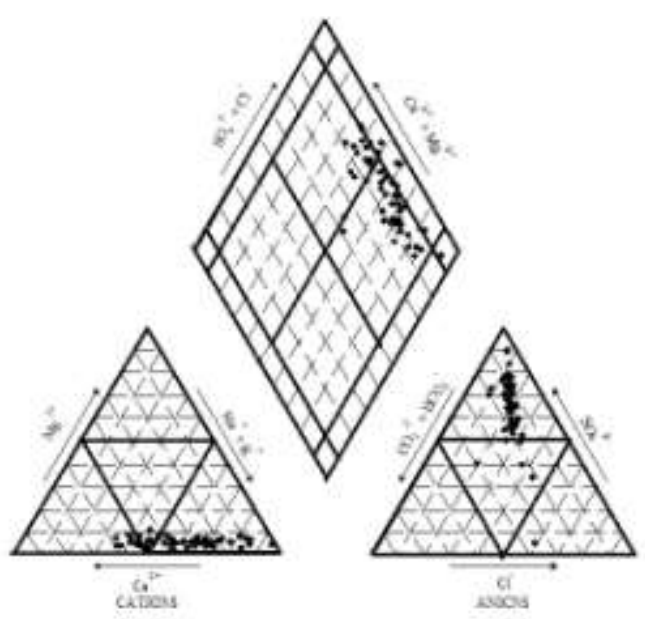

(b. Post-monsoon season)

Fig. 3.a and b:PiperTrilinear diagram showing analysis of dug well water samples of the study area.

\subsection{Chemistry of Groundwater based on Gibbs Variation Diagram}

Gibbs variation diagram is useful to understand the chemistry of groundwater [12].

It is seen from the Fig. 4.a that out of 56 dug well water samples of pre-monsoon season, 26 dug well water samples

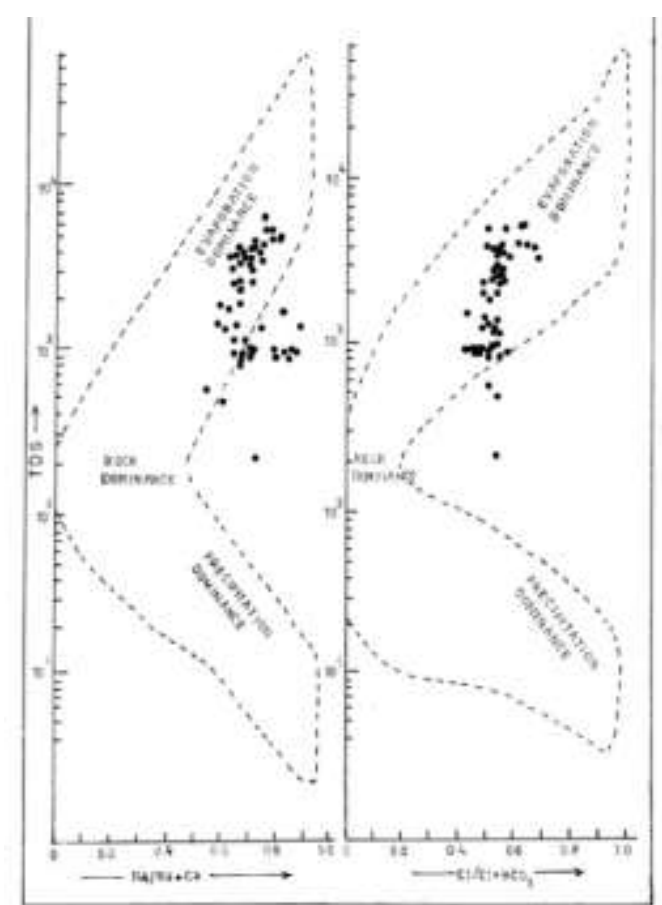

(a. Pre-monsoon season)
(46\%) belongs to evaporation dominance and 30 dug well water samples (54\%) suggests rock dominance.

From the Fig. 4.b,it is observed that out of 56 dug well water samples of post-monsoon season, 36 dug well water samples (64\%) represents evaporation dominance and 20 dug well water samples $(36 \%)$ suggests rock dominance.

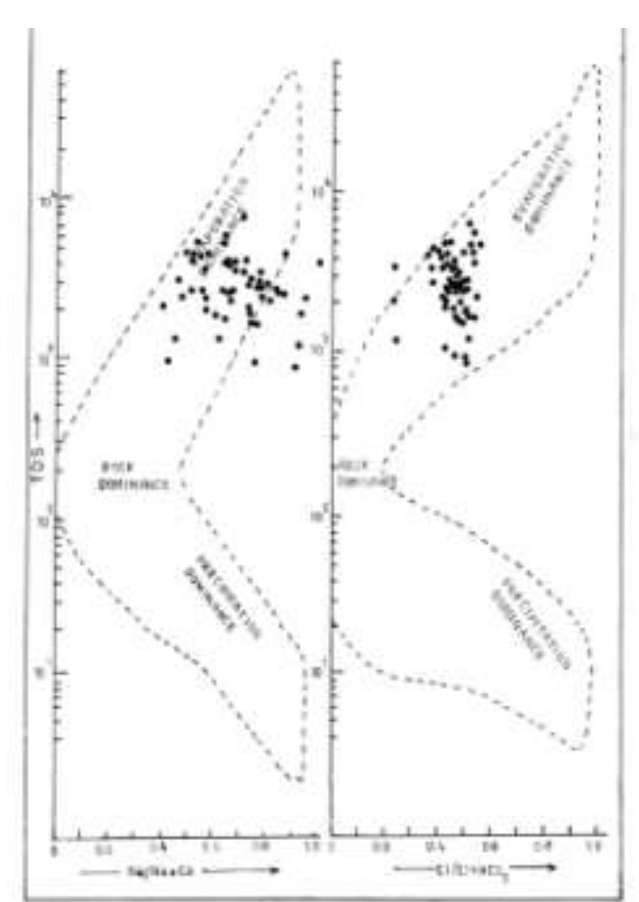

(b. Post-monsoon season)

Fig.4.a and b: Gibbs Variation diagram of dug well water quality of the study area. 


\section{CONCLUSION}

The analytical results shows $72 \%$ and $95 \%$ of dug well water samples having higher concentration of EC values in pre-monsoon and post-monsoon seasons respectively. The TDS values of $98 \%$ dug well water samples in pre-monsoon and $95 \%$ in post-monsoon season are with higher concentrations. Similarly, Ca values are higher in $63 \%$ and $72 \%$ dug well water samples of pre and post-monsoon seasons respectively. The concentration of Total hardness in pre and post-monsoon seasons in dug well water samples are $96 \%$ and $100 \%$ in pre and post-monsoon seasons. Similarly $\mathrm{Na}, \mathrm{SO}_{4}$ andCl also shows higher concentration, which indicates signs of deterioration of water quality as per WHO and ISI standards. The proper drainage system is required where electrical conductivity (EC) is more than $1500 \mu \mathrm{s} / \mathrm{cm}$.A few dug well water samples the values of electrical conductivity and TDS are higher because of the application of heavy fertilizers for agricultural practices.

The Physico-chemical analyses of dug well water samples represents $\mathrm{NA}+\mathrm{K}>\mathrm{Ca}+\mathrm{Mg}$ (Alkali exceed alkaline earth) hydro chemical facies and $\mathrm{Cl}+\mathrm{SO}_{4}>\mathrm{HCO}_{3}+\mathrm{CO}_{3}$ ( Strong acids exceed weak acids ) hydro chemical facies. The Chemistry of ground water belongs to evaporation dominance $(46 \%)$ and rock dominance $(54 \%)$ of pre monsoon season and evaporation dominance $(64 \%)$ and rock dominance $(36 \%)$ of post monsoon season.

These chemical analysis of dug well water samples reveals that $98 \%$ groundwater samples of pre- monsoon season and $70 \%$ groundwater samples of post-monsoon season represent $\mathrm{Na}+\mathrm{K}>\mathrm{Ca}+\mathrm{Mg}$ (alkalies exceed alkaline earth) hydrochemicalfacies. Similarly, $100 \%$ groundwater samples of pre-monsoon season and $84 \%$ groundwater samples of post-monsoon season suggests $\mathrm{Cl}+\mathrm{SO}_{4}>\mathrm{HCO}_{3}+\mathrm{CO}_{3}$ (strong acids exceed weak acids) hydrochemicalfacies. The $2 \%$ and $30 \%$ groundwater samples of pre-monsoon and post-monsoon seasons suggests $\mathrm{Ca}+\mathrm{Mg}>\mathrm{Na}+\mathrm{K}$ (alkaline earths exceed alkalies) hydrochemicalfacies. The $16 \%$ groundwater samples of post-monsoon season represent $\mathrm{Cl}+\mathrm{SO}_{4}$ (strong acids) hydrochemicalfacies.

Gibbs variation diagram suggests the chemistry of groundwater samples belongs to evaporation dominance $(46 \%)$ and rock dominance $(54 \%)$ in pre-monsoon season and evaporation dominance $(64 \%)$ and rock dominance $(36 \%)$ in post-monsoon season.

\section{REFERENCES}

[1]. Panaskar, D.B., Yedekar, D.B. and Deshpande, S.M. (2007):Assessment of groundwater quality of Nanded city,Maharashtra, Gondwana Geological Magazine, Vol.11, pp.77-86.

[2]. Yadav, A. S., Sawant, P. T., Pishte, J. B. and Sajane, A.S. (2011):Hydrogeological and groundwater quality studies ofJaysingpur town, Kolhapur district, Maharashtra, Env. Poll. Control Jour.Vol. 14 (6), pp. 71-76.

[3]. Krishna Anantha, Loganathan K. and Ahamed A. Jafar (2012): Study of groundwater quality and its suitability fordrinking purpose in Alathur block, Perambalpur District, Archives of Applied Science Research, vol.4(3), pp.132138.

[4]. Deshpande, S. M. and Aher, K.R.(2012): Evaluation of groundwater quality and its suitability for drinking andagricultural uses in parts of Vijapur, Aurangabad District, Maharashtra, India, Research Jr. of Chemical Sciences, vol.1, pp.25-31.

[5]. Narsimha A. and Anitha(2013): Evaluation of groundwater quality and its suitability for drinking purposes in Gunthakal area, Anantpur District, A.P. , India, vol.4,No. 2, pp. 70-76.

[6]. Pisal, P. A. and Yadav, A. S.(2014): Groundwater Quality Assessment of Bhogavati River Basin, Kolhapur District, Maharashtra, India, Int. Jr. of Surface and Groundwater Mgt., Vol.1, No. 2, pp.83-89.

[7]. WHO, World Health Organization, (2004): Guideline for Drinking Water Quality, $3^{\text {rd }}$ Edition (Recommendation), World Health Organization, Geneva.

[8]. ISI, Indian Standards Institution,(1983) : Indian Standards Specifications for drinkingwater,Pu.No. IS10500-1983,Indian Standard Institution, New Delhi.

[9]. APHA, AWWA, WPCF, (1992): Standard Methods For the Examination of Water and Waste water, $\left(19^{\text {th }}\right.$ Edn.),American Public Health Association, Washington D.C.

[10]. U.S. Salinity Laboratory Staff (1954): Diagnosis and improvement of saline and alkali soils, U. S. Dept. of Agriculture Hand Book, No.60, $2^{\text {nd }}$ Edn.

[11]. Piper, A. M. (1953): A Graphic procedure in the geochemical interpretation of water analysis, U. S. Geol. Surv.Groundwater Note 12, pp. 50-59.

[12]. Gibbs, R.J., (1970): Mechanisms Controlling World's Water Chemistry, Science, Vol.170, pp.1088-1090. 